\title{
Latest Advances for the Treatment of Chronic Plaque Psoriasis with Biologics and Oral Small Molecules
}

\section{Francesco Bellinato \\ Paolo Gisondi iD Giampiero Girolomoni (i) \\ Department of Medicine, Section of Dermatology and Venereology, University of Verona, Verona, Italy}

\begin{abstract}
Psoriasis is a common immune-mediated chronic skin disease. Disease severity is influenced by several factors including the extent and localization of skin lesions, severity of pruritus and comorbidities, such as psoriatic arthritis. Moderate to severe psoriasis is defined when cutaneous involvement is diffuse, covering more than $10 \%$ of the body surface areas and/or involving the sensitive areas such as face, genitalia, folds or nails or has high impact on patients' quality of life, and it occurs in approximately $15 \%$ of cases. In recent years, a growing understanding of psoriasis pathophysiology allowed the development of an increasing number of effective and safe treatments, including biologicals that are indicated for moderate to severe psoriasis. Different classes of biologicals have been already approved, including TNF- $\alpha$ (etanercept, infliximab, adalimumab and certolizumab pegol), IL-12/23 (ustekinumab), IL-17 (secukinumab, ixekizumab, brodalumab) and IL-23 (guselkumab, risankizumab, tildrakizumab) inhibitors. The objective of this narrative review is to revise efficacy and safety data of the latest biologicals, small oral molecules and biosimilar drugs for the treatment of chronic plaque psoriasis at Phase III of clinical development. The latest IL-17 and IL-23 inhibitors include bimekizumab, netakimab and mirikizumab as well as oral small molecules, such as deucravacitinib, a tyrosine kinase 2 selective inhibitor, and piclidenoson, an agonist of the Gi protein-associated A3 adenosine receptor. Additional molecules are in an early phase of development. Highly promising biologicals and small oral molecules are the leading edge of the systemic treatment of psoriasis.
\end{abstract}

Keywords: psoriasis, bimekizumab, mirikizumab, netakimab, sonelokimab, deucravacitinib, piclidenoson, biosimilars, biologics

\section{Introduction}

Psoriasis is a chronic, immuno-mediated skin inflammatory disease frequently affecting $2-3 \%$ of general population. ${ }^{1}$ Disease severity is influenced by several factors including the extent and localization of skin lesions and comorbidities, such as psoriatic arthritis, as well as the impact on life (Figure 1A). ${ }^{2}$ The treatment success entails the achievement of psoriasis clearance or almost clearance, which best correlates with improvement in quality of life (Figure 1B). Over the last years, highly effective targeted therapies have been developed, including conventional, biologicals and oral small molecules. The classes of biologicals include tumor necrosis factor (TNF)- $\alpha$ inhibitors (etanercept, infliximab, adalimumab and certolizumab pegol), the interleukin (IL)-12/23 inhibitor (ustekinumab), the IL-17 inhibitors (secukinumab, ixekizumab, and brodalumab) and IL-23 inhibitors
Correspondence: Francesco Bellinato Department of Medicine, Section of Dermatology and Venereology, University of Verona, Verona, Italy

Email francesco.bellinato@univr.it 

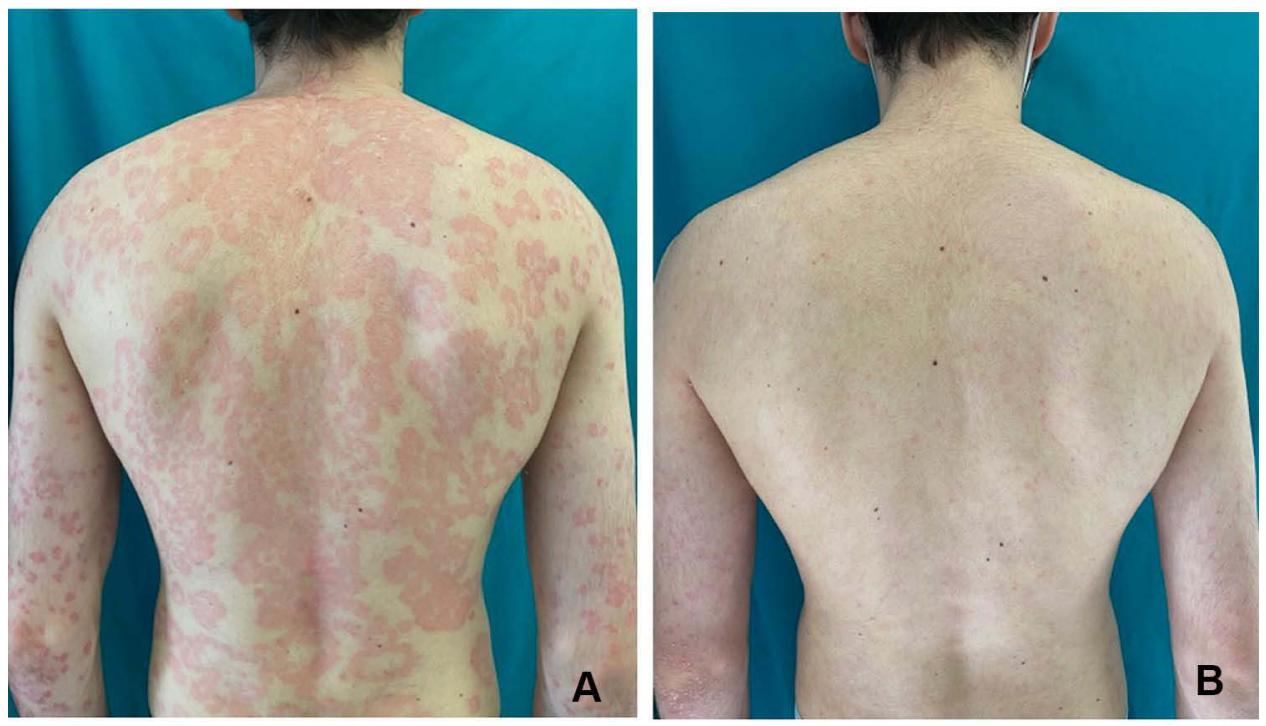

Figure I A 27-year-old man affected by moderate to severe psoriasis presenting with erythematous-desquamative plaques on the back (A). Complete cutaneous clearance after 12 weeks treatment with an IL-23 inhibitor (B).

(guselkumab, tildrakizumab, and risankizumab). ${ }^{3}$ The advent of new biologicals has raised the standard of efficacy and safety in the treatment of psoriasis, even if there is still a medical need for novel treatments allowing a higher cutaneous clearance and durability. ${ }^{4,5}$ In addition, most treatments lose efficacy over time, thus with new treatment options needed. In recent years, a growing understanding of psoriasis pathophysiology allowed the development of an increasing number of effective and safe treatments. In this narrative review, latest development with biological, small oral molecules and new biosimilar drugs for the treatment of chronic plaque psoriasis are discussed.

\section{Materials and Methods}

A narrative review based on electronic searches either on PubMed $^{\circledR}$ or clinicaltrials.org database was performed. Original articles investigating emerging biological, small oral molecules and new biosimilars for chronic plaque psoriasis at phase III stage of clinical development were retrieved. Search strategy in clinicaltrials.org included all the phase III interventional studies reporting results or in recruiting phase. Secondly, consecutive searches on PubMed $^{\circledR}$ using "bimekizumab", "UCB4940", "netakimab", "BCD-085", "mirikizumab", "LY3074828”, “sonelokimab", "IL17MS3086", "deucravacitinib", "BMS986165", "picledenoson", "CF101”, biosimilars and psoriasis as search term were performed. References of all the identified original research articles were also checked for any additional literature. For all the drugs included, data concerning mechanisms, pharmaceutical company, currently investigated indications, efficacy and safety profile were collected (Table 1). Emerging drugs in Phase II of clinical development have been described shortly in the discussion.

\section{Results}

\section{IL-I 7 Inhibitors Bimekizumab}

Bimekizumab is a monoclonal humanized IgG1 antibody that blocks IL-17F and IL-17A developed by UCB. ${ }^{6}$ Bimekizumab differs from secukinumab and ixekizumab, which are selective inhibitors of IL-17A and from brodalumab which is an IL-17 receptor inhibitor. ${ }^{6}$ The additive advantage of dual IL-17A and F is being investigated. ${ }^{7}$ Initial phase II studies showed that bimekizumab allows rapid and durable clinical improvements in patients with moderate to severe psoriasis with an expected safety profile. $^{8}$ The results of two phase III clinical trials of bimekizumab, BE VIVID and BE READY, have recently been published. ${ }^{9,10}$ BE VIVID is a multicenter, doubleblind, controlled trial comparing the efficacy and safety of bimekizumab versus placebo and ustekinumab over 52 weeks. At 16 weeks, the PASI90 was achieved by $85 \%$ of the patients on bimekizumab versus $50 \%$ on ustekinumab versus $5 \%$ on placebo. Investigator global assessment (IGA) score of $0-1$ (clear and almost clear skin) was achieved by $84 \%$ of patients on bimekizumab versus 
Table I Latest Biologics and Oral Small Molecules for the Treatment of Chronic Plaque Psoriasis on Clinical Development

\begin{tabular}{|l|l|l|l|l|l|}
\hline Drug & Target & Type of Molecule & $\begin{array}{l}\text { PASI75 (Drugs vs } \\
\text { Placebo) }\end{array}$ & $\begin{array}{l}\text { PASI90 (Drugs vs } \\
\text { Placebo) }\end{array}$ & $\begin{array}{l}\text { PASI I00 (Drugs vs } \\
\text { Placebo) }\end{array}$ \\
\hline $\begin{array}{l}\text { Bimekizumab } \\
\text { (UCB4940) }\end{array}$ & $\begin{array}{l}\text { IL-I7A and } \\
\text { IL-I7F }\end{array}$ & $\begin{array}{l}\text { Humanized IgGI monoclonal } \\
\text { antibody }\end{array}$ & $95 \%$ vs I\% at week I6 & $\begin{array}{l}85-91 \% \text { vs I\% at } \\
\text { week } 16\end{array}$ & $\begin{array}{l}59-68 \% \text { vs } 5 \% \text { at } \\
\text { week } 16\end{array}$ \\
\hline $\begin{array}{l}\text { Mirikizumab } \\
\text { (LY3074828) }\end{array}$ & $\begin{array}{l}\text { PI9 subunit } \\
\text { of IL-23 }\end{array}$ & $\begin{array}{l}\text { Humanized IgG4 monoclonal } \\
\text { antibody }\end{array}$ & $90 \%$ vs $8 \%$ at week 16 & $\begin{array}{l}74 \% \text { vs } 6 \% \text { at week } \\
16\end{array}$ & $32 \%$ vs I\% at week 16 \\
\hline $\begin{array}{l}\text { Netakimab } \\
\text { (BCD085) }\end{array}$ & IL-17A & $\begin{array}{l}\text { Humanized IgGI monoclonal } \\
\text { antibody }\end{array}$ & $\begin{array}{l}83 \% \text { vs II\% at week } \\
24\end{array}$ & $\begin{array}{l}68 \% \text { vs } 7 \% \text { at week } \\
24\end{array}$ & $49 \%$ vs $7 \%$ at week 24 \\
\hline $\begin{array}{l}\text { Deucravacitinib } \\
\text { (BMS-986I65) }\end{array}$ & TYK2 & Oral TYK2 selective inhibitor & $\begin{array}{l}75 \% \text { vs } 7 \% \text { at week } 12 \\
(\text { phase II) }\end{array}$ & Not available & Not available \\
\hline $\begin{array}{l}\text { Piclidenoson } \\
\text { (CFI0I) }\end{array}$ & A3AR & $\begin{array}{l}\text { Oral agonist of the Gi protein } \\
\text { associated A3AR }\end{array}$ & Not available & Not available & Not available \\
\hline
\end{tabular}

Abbreviations: TYK2, tyrosine kinase 2 selective inhibitor; A3AR, A3 adenosine receptor (A3AR).

$53 \%$ of those on ustekinumab and $5 \%$ on placebo. Complete clearance at week $16(\mathrm{PASI} 100$ or IGA=0) was reached by $59 \%$ of the patients in the bimekizumab arm compared to $21 \%$ of patients in the ustekinumab arm. ${ }^{9} \mathrm{BE}$ READY is a multicenter, double-blind, placebo-controlled, randomized trial investigating the effects of bimekizumab withdrawal and two maintenance dosing schedules (320 mg every 4 versus 8 weeks) over 56 weeks. PASI90 was achieved by $91 \%$ of the patients on bimekizumab compared to $1 \%$ of those on placebo. IGA score of $0-1$ was achieved by $93 \%$ on bimekizumab versus $1 \%$ of those on placebo. A sustained cutaneous clearance was maintained after drug withdrawal with a median time to loss of PASI75 of 32 weeks. ${ }^{10}$ Finally, the results of the two Phase 3 , multicenter, randomized, double-blind, active comparator, controlled trial BE SURE (NCT03412747) and BE RADIANT (NCT03536884) are available. ${ }^{11,12}$ Bimekizumab was compared either to adalimumab or secukinumab, respectively. BE SURE showed that at week 16 , PASI 90 response was achieved by $86.2 \%$ of the patients treated with bimekizumab, compared to $47.2 \%$ who received adalimumab. BE RADIANT showed that at week 48, PASI 100 was achieved by $67.0 \%$ of the patients treated with bimekizumab, compared to $46.2 \%$ of the treated with secukinumab. In all these studies, the safety profile was consistent with that observed in the previous phase IIb study: treatment emergent adverse events (TEAEs) occurred in $61 \%$ of bimekizumab treated patients versus $36 \%$ of the placebo arm, more commonly nasopharyngitis and upper respiratory tract infections (URTI). Oral candidiasis occurred in $19 \%$ of the patients treated with bimekizumab versus $3 \%$ of those on secukinumab. ${ }^{12}$ Only one out of 744 bimekizumab treated patients in both BE VIVID and BE READY studies developed inflammatory bowel disease and there was one case of new-onset ulcerative colitis either in the bimekizumab or secukinumab group. ${ }^{9,10,12}$ A placebo-controlled randomized proof-ofconcept clinical trial investigated safety, pharmacokinetics and clinical efficacy of bimekizumab at multiple doses (range $560 \mathrm{mg} / 320 \mathrm{mg} / 320 \mathrm{mg}-80 \mathrm{mg} / 40 \mathrm{mg} / 40 \mathrm{mg}$ ) for psoriatic arthritis. At week 8, American College of Rheumatology 20\% (ACR20) response was achieved by $80 \%$ of the patients treated with top three doses bimekizumab compared to $17 \%$ in the placebo arm. No unexpected safety signals were reported. ${ }^{9}$ There are ongoing phase II clinical trials to test the efficacy of bimekizumab in the treatment of hidradenitis suppurativa (NCT03248531).

\section{Netakimab}

Netakimab is an injectable humanized IgG1 nanobody that targets IL-17A developed by BIOCAD. In netakimab, the VH domain is replaced by the VHH domain of Lama possessing a long complementarity determining region (CDR-H3) in its heavy chain that has a high affinity to IL-17A. ${ }^{13}$ Nanobodies are a new generation drug based on single-domain of heavy-chain-only antibodies exhibiting higher stability and higher penetration in the tissues. Netakimab is currently registered in Russia $\left(\right.$ Efleira $^{\circledR}$ ) for the treatment of moderate-to-severe 
psoriasis in adults. ${ }^{14}$ Initial phase II trials showed that 93\% of the subjects achieved PASI75 within 3 months and netakimab maintained efficacy, favorable safety profile and low immunogenicity during one year of the treatment. Preliminary results of BCD-085-7/PLANETA study (NCT03390101), a comparative, randomized, double-blind, placebo-controlled phase III clinical study, showed that at week 12 PASI75 was achieved by $83.3 \%$ and $77.7 \%$ of the patients treated with netakimab $120 \mathrm{mg}$ every four and every two weeks, respectively, compared to $0 \%$ in placebo arm. ${ }^{15-17}$ The drug showed a favorable safety profile consistent with those expected for the class of IL-17 inhibitors, and low immunogenicity. ${ }^{15}$ Finally, the results of the 24-week period phase III clinical trial (PATERA study) investigating the efficacy and safety of netakimab compared to placebo in patients with active psoriatic arthritis showed that netakimab $120 \mathrm{mg}$ is significantly superior to placebo in patients with active psoriatic arthritis. In this study PASI75, PASI90 and PASI100 were reported as secondary endpoints (Table 1). ${ }^{18}$

\section{IL-23 Inhibitors Mirikizumab}

Mirikizumab is a humanized monoclonal IgG4 variant antibody targeting the p19 subunit of IL-23 developed by Eli Lilly. ${ }^{19}$ Mirikizumab was initially investigated in 2019 in a phase II randomized controlled trial comparing different doses $(30,100$, or $300 \mathrm{mg})$ given at 0 and 8 weeks. PASI90 was achieved by $59 \%$ and $67 \%$ of the patients treated with mirikizumab $100 \mathrm{mg}$ and $300 \mathrm{mg}$, respectively. Results of the phase III randomized, double-blind, placebo-controlled study OASIS-1 (NCT03482011) showed that $64 \%$ of the patients treated with mirikizumab $250 \mathrm{mg}$ every 4 weeks achieved PASI90 at 16 weeks versus $6.5 \%$ in the placebo arm and $69 \%$ achieved IGA $0-1$ compared to $6.5 \%$ in the placebo arm. ${ }^{20}$ The preliminary results of OASIS-2 (NCT03535194), a multicenter randomized, double-blind, placebo-controlled study comparing the efficacy and safety of mirikizumab to placebo and secukinumab, showed that mirikizumab met the primary and all key secondary endpoints versus placebo at week 16 (superiority) and all key secondary endpoints versus the active comparator at week 16 (non-inferiority) and week 52 (superiority). ${ }^{21}$ Mirikizumab is currently being investigated in the phase III study OASIS- $3 .^{22}$ The percentage of patients reporting at least one AE was similar among patients treated with placebo or mirikizumab.
The most common were viral infections, hypertension, infections of the respiratory tract, injection-site pain and diarrhoea. ${ }^{15}$ Despite these promising results, Eli Lilly no longer plans to submit mirikizumab for regulatory approval in psoriasis and will prioritize on other indications. Indeed, there are ongoing phase II clinical trials to test mirikizumab in the treatment of ulcerative colitis and Crohn's disease. ${ }^{23}$

\section{Sonelokimab}

Sonelokimab (IL17MS3086) is a trivalent camelid nanobody binding to IL-17A and F at Phase 2 stage, developed by Merck Serono in collaboration with Avillion. Camelid antibodies derived from the Camelidae family of mammals, including llamas, camels, and alpacas are characterized by heavy-chain-only structure and have the advantages of small proteins with the benefits of monoclonal antibody properties. Initial results showed that $76.5 \%$ of the patients treated with sonelokimab $120 \mathrm{mg}$ (augmented load) achieved PASI 90 by week 12. The safety profile was consistent with IL-17 inhibitors, with Candida infections observed in $17.4 \%$ of patients. ${ }^{24}$

\section{Oral Small Molecules Deucravacitinib}

Deucravacitinib (BMS-986165) is an oral Tyrosine kinase 2 (TYK2) selective inhibitor developed by Bristol-Myers Squibb for the treatment of moderate to severe chronic plaque psoriasis and psoriatic arthritis. In contrast to other Janus kinase (JAK), which act as ATP competitor, deucravacitinib binds to the regulatory domain of TYK2, this providing a more selective activity. Deucravacitinib inhibits IL-12, IL-23 and type 1 interferon pathways, which are implicated in the pathogenesis of psoriasis and other immune-mediated diseases. ${ }^{25}$ The results of the Phase II, randomized, double-blinded, placebo-controlled study in five different deucravacitinib dosing groups are promising. At week 12, the PASI75 was achieved by $7 \%$ of the patients in the placebo arm versus $75 \%$ with deucravacitinib $12 \mathrm{mg}$ daily. AEs were reported in 55-80\% of intervention groups compared to $51 \%$ in the placebo. Most common AEs included nasopharyngitis, headache, diarrhea, nausea and URTIs and acne was found more frequently in the deucravacitinib group. ${ }^{26}$ Deucravacitinib is currently investigated in moderate to severe plaque psoriasis in different phase III clinical trials versus placebo (POETYK PSO-LTE NCT04036435, POETYK PSO-3 NCT04167462) and versus apremilast (POETYK PSO-1 NCT03624127, POETYK- 
PSO-2 NCT03611751); in adolescent and Japanese patients (NCT04772079, NCT04772079).

\section{Piclidenoson}

Piclidenoson (CF101) is an oral agonist of the Gi protein associated $\mathrm{A} 3$ adenosine receptor $\left(\mathrm{A}_{3} \mathrm{AR}\right)$ developed by Can-Fite BioPharma. $A_{3}$ AR is highly expressed in inflammatory skin conditions and in the peripheral blood mononuclear cells. The anti-inflammatory effects may be mediated by the downregulation of the NF- $\mathrm{kB}$ signaling pathway, and in turn TNF- $\alpha$ inhibition. ${ }^{27}$ The efficacy of piclidenoson has been already demonstrated in phase II clinical studies. Results of a phase II, multicenter, doubleblinded, placebo control trial involving 75 patients randomized to placebo, 1-, 2- or $4 \mathrm{mg}$ piclidenoson twice a day showed that only the $2 \mathrm{mg}$ group achieved improvement vs placebo in mean change of PASI at weeks 8 and 12; in particular, PASI50 was achieved by $35.3 \%$ of the 17 patients treated with $2 \mathrm{mg}$ dose. AEs incidence in the 1-, 2- and $4 \mathrm{mg}$ arm was $58.3 \%, 17.6 \%$ and $13.3 \%$, respectively, compared to $21.1 \%$ in the placebo arm. Except one severe psoriasis exacerbation, all AEs were mild/ moderate. ${ }^{28}$ Piclidenoson is currently investigated in a phase III randomized, double-blind, placebo- and active comparator (apremilast)-controlled trial (NCT03168256), but the results are not available yet.

\section{Discussion}

Psoriasis has significant and cumulative impacts on physical and psychological morbidity and health-related quality of life. ${ }^{29}$ A plethora of comorbid conditions are associated with moderate to severe psoriasis, sharing genetic predisposition and inflammatory pathways. Cardiovascular and metabolic diseases are particularly important because can potentially reduce life expectancy of the patients. ${ }^{30}$ Significant advancement in the treatment of psoriasis has been gained over the past 30 years. The advent of new medications with unique mechanisms of action affords significant opportunities for better disease control with an acceptable safety profile. Over the last 15 years, new biologicals raised progressively the therapeutic targets, from PASI 75 to PASI 90 and to absolute PASI less than $3 .^{31}$ Biologicals differ in their efficacy towards cutaneous disease and joint disease as well as in their safety profiles. According to a recent network analyses with head-to-head comparisons, anti-IL-17 and anti-IL-23 agents demonstrate higher cutaneous response rates than anti-TNF- $\alpha$ therapies and anti-IL $-12 / 23$ across multiple endpoints and over short-term and long-term follow-up. ${ }^{32}$ Moreover, TNF- $\alpha$ and IL-17 inhibitors represent a first line biological treatment for psoriatic arthritis, particularly in case of involvement of the axial, tendons and entheses domains. ${ }^{33}$ Apart from piclidenoson and deucravacitinib, other oral molecules are currently being investigated in phase II clinical trials, but their place in therapy is difficult to predict. Vimirogant (VTP-43742) is a highly potent, selective, oral retinoic acid receptor-related orphan receptor gamma $\mathrm{T}(\mathrm{ROR} \gamma \mathrm{T})$ inverse agonist developed by Vitae Pharmaceuticals. ROR $\gamma$ T is required for Th17 differentiation and expression of IL-17A, IL-17F, IL-22, IL26 and IL-23R. The results of the phase II, randomized, double-blind, placebo-controlled trial assessing Vimirogant at multiple (350 mg and $700 \mathrm{mg}$ ) oral doses in patients with moderate to severe psoriasis over a four-week period showed clinically relevant and statistically significant reductions in PASI score relative to baseline values. Over this period, a $30 \%$ reduction in PASI score relative to placebo was achieved by patients receiving $700 \mathrm{mg}$ dose. ${ }^{34}$ Vimirogant was well tolerated, the most reported AEs were mild, mainly headache and nausea. ${ }^{35}$ Transaminase elevations were observed in four treated with the $700 \mathrm{mg}$ dose group. ${ }^{34}$ Ponesimod, a sphingosine-1-phosphate receptor (S1PR1) antagonist developed by Actelion. Ponesimod blocks $\mathrm{T}$ cells in the secondary lymphoid tissue and prevents their infiltration in the target organs such as the skin. This drug is burdened by the rapid loss of efficacy when discontinued and by cardiologic and pneumological AEs. ${ }^{36}$ The competition between the high standard of efficacy and safety of the new monoclonal antibodies, as well as biosimilars, may limit small oral molecule space in the landscape of treatment for psoriasis. Nonetheless, the advantages provided by the route of administration may be particularly suitable for patients suffering from trypanophobia. ${ }^{37}$ In the recent years, different anti-TNF- $\alpha$ biosimilars allowed the dermatologist to prescribe effective biologics at a very competitive cost. ${ }^{38,39}$ Biosimilars are medications very similar to the originator approved biologic without clinically significative safety, purity, and potency differences and different only in terms of the manufacturing process, excipient and delivery device used. The biosimilars are tested versus originators through doubleblind crossover design trials assessing pharmacokinetics, immunogenicity and drug safety. ${ }^{40}$ Currently, several antiTNF- $\alpha$ biosimilars approved by regulatory authorities are available and other molecules (eg, ustekinumab) are being investigated in clinical trials.

Although the review is updated to the most recent available results of the clinical trials, we acknowledge 
that the current study may be limited given its not systematic design and because it is not assessing drugs for psoriatic arthritis.

\section{Conclusion}

Highly promising biologicals and small oral molecules are in the pipeline of the treatment of psoriasis. The systemic treatment armamentarium for psoriasis is expanding with very effective and safe drugs including emerging biosimilars that may offer an economic solution for patients without compromising on efficacy.

\section{Abbreviations}

$\mathrm{A}_{3} \mathrm{AR}, \mathrm{A} 3$ adenosine receptor; ACR, American College of Rheumatology 20\%; AE, adverse event; IGA, Investigator global assessment; KAK, Janus kinase; PASI, Psoriasis area and severity index; ROR $\gamma \mathrm{T}$, receptor-related orphan receptor gamma T; sPGA, static Physician's Global Assessment; S1PR1, sphingosine-1-phosphate receptor; TYK, tyrosine kinase 2; URTI, upper respiratory tract infections.

\section{Funding}

The study was supported by Fondazione Cariplo, Fondazione Veronesi, Impact of COVID19 infection on patients affected by inflammatory skin diseases on immuno-suppressive therapies (COVISKIN); ID 1833073 rif. 2020-1363.

\section{Disclosure}

Francesco Bellinato has nothing to declare. Paolo Gisondi received consultation fee from AbbVie, Amgen, Almirall, Eli Lilly, Janssen, Leo Pharma, Novartis, Sanofi, Sandoz, UCB as a speaker and/or participants in advisory boards. Giampiero Girolomoni served as consultant and/or speaker for AbbVie, Abiogen, Almirall, Amgen, Biogen, Boehringer-Ingelheim, Bristol-Meyers Squibb, Celltrion, Eli-Lilly, Genzyme, Leo Pharma, Menlo therapeutics, Novartis, Pfizer, Regeneron, Samsung, Sandoz, Sanofi and UCB. The authors report no other conflicts of interest in this work.

\section{References}

1. Greb JE, Goldminz AM, Elder JT, et al. Psoriasis. Nat Rev Dis Primers. 2016;2:16082. doi:10.1038/nrdp.2016.82

2. Gisondi P, Altomare G, Ayala F, et al. Italian guidelines on the systemic treatments of moderate-to-severe plaque psoriasis. $J$ Eur Acad Dermatol Venereol. 2017;31(5):774-790. doi:10.1111/jdv.14114
3. Ghoreschi K, Balato A, Enerbäck C, Sabat R. Therapeutics targeting the IL-23 and IL-17 pathway in psoriasis. Lancet. 2021;397:754-756. doi:10.1016/S0140-6736(21)00184-7

4. Balogh EA, Bashyam AM, Ghamrawi RI, Feldman SR. Emerging systemic drugs in the treatment of plaque psoriasis. Expert Opin Emerg Drugs. 2020;25:89-100. doi:10.1080/14728214.2020.1745773

5. Kimball AB, Leonardi C, Stahle M, et al. Demography, baseline disease characteristics and treatment history of patients with psoriasis enrolled in a multicentre, prospective, disease-based registry (PSOLAR). $B r \quad J$ Dermatol. 2014;171:137-147. doi:10.1111/ bjd. 13013

6. Bertelsen T, Ljungberg C, Boye Kjellerup R, Iversen L, Johansen C. IL-17F regulates psoriasis-associated genes through IkappaBzeta. Exp Dermatol. 2017;26:234-241. doi:10.1111/exd.13182

7. Adams R, Maroof A, Baker T, et al. Bimekizumab, a novel humanized IgG1 antibody that neutralizes both IL-17A and IL-17F. Front Immunol. 2020;11:1894. doi:10.3389/fimmu.2020.01894

8. Papp KA, Merola JF, Gottlieb AB, et al. Dual neutralization of both interleukin $17 \mathrm{~A}$ and interleukin $17 \mathrm{~F}$ with bimekizumab in patients with psoriasis: results from BE ABLE 1, a 12-week randomized, double-blinded, placebo-controlled phase $2 \mathrm{~b}$ trial. $J$ Am Acad Dermatol. 2018;79:277-86.e10. doi:10.1016/j.jaad.2018.03.037

9. Reich K, Papp KA, Blauvelt A, et al. Bimekizumab versus ustekinumab for the treatment of moderate to severe plaque psoriasis (BE VIVID): efficacy and safety from a 52-week, multicentre, double-blind, active comparator and placebo controlled phase 3 trial. Lancet. 2021;397:487-498. doi:10.1016/S0140-6736(21)00125-2

10. Gordon KB, Foley P, Krueger JG, et al. Bimekizumab efficacy and safety in moderate to severe plaque psoriasis (BE READY): a multicentre, double-blind, placebo-controlled, randomised withdrawal phase 3 trial. Lancet. 2021;397:475-486. doi:10.1016/S01406736(21)00126-4

11. Warren RB, Blauvelt A, Bagel J, et al. Bimekizumab versus adalimumab in plaque psoriasis. N Engl J Med. 2021;23.

12. Glatt S, Baeten D, Baker T, et al. Dual IL-17A and IL-17F neutralisation by bimekizumab in psoriatic arthritis: evidence from preclinical experiments and a randomised placebo-controlled clinical trial that IL-17F contributes to human chronic tissue inflammation. Ann Rheum Dis. 2018;77:523-532. doi:10.1136/annrheumdis-2017212127

13. Reichert J. Anti-IL17 netakimab registered in Russia antibodysociety. org: antibody Society; 2019. Available from: https://www.antibodyso ciety.org/antibody-therapeutic/anti-il17-netakimab-registered-in-rus sia. Accessed June 15, 2021.

14. BIOCAD registered the first Russian original therapeutic monoclonal antibody: BIOCAD biotechnology company; 2019. Available from: https://biocadglobal.com/post/Russian_original_therapeutic_monoclo nal_antibody. Accessed December 29, 2019.

15. Kubanov AABA, Samtsov AV, Khairutdinov VR, et al. Netakimab new IL17a inhibitor: 12-week results of phase III clinical study BCD085-7/PLANETA in patients with moderate-to-severe plaque psoriasis. Vestnik Dermatologii Ivenerologii. 2019;95:15-28.

16. Bakulev AL, Samtsov AV, Kubanov AA, Khairutdinov VR, Kokhan MM, Artemyeva AV. Long-term efficacy and safety of netakimab in patients with moderate-to-severe psoriasis. Results of phase II open-label extension clinical study BCD-085-2-ext. Vestnik Dermatologii Ivenerologii. 2019;95:56-64.

17. Bakulev A, Samtsov A, Artemeva A, et al. Netakimab: 12-week results from planeta study, a phase iii trial of a novel IL-17 inhibitor in moderate-to-severe plaque psoriasis. In: Abstract of the 24th World Congress of Dermatology (WCD) in Milan, 10-15 June 2019.

18. Korotaeva TV, Mazurov VI, Lila AM, et al. The efficacy and safety of netakimab in patients with psoriatic arthritis: results from the Phase III clinical study PATERA. Nauchcno-Practicheskaya Revmatologia. 2020;58:480-488. doi:10.47360/1995-4484-2020$480-488$ 
19. Reich K, Rich P, Maari C, et al. Efficacy and safety of mirikizumab (LY3074828) in the treatment of moderate-to-severe plaque psoriasis: results from a randomized phase II study. $\mathrm{Br} J$ Dermatol. 2019;181:88-95. doi:10.1111/bjd.17628

20. ClinicalTrials.gov [Internet]. 2000 Feb 29 - Identifier NCT03482011, A study to evaluate the efficacy and safety of mirikizumab (LY3074828) in participants with moderate-to-severe plaque psoriasis (OASIS-1). Bethesda (MD): National Library of Medicine (US); 2006. Available from: https://clinicaltrials.gov/ct2/show/ NCT03482011 ?term=OASIS-1+mirikizumab\&draw=2\&rank=1. Accessed April 26, 2020.

21. ClinicalTrials.gov [Internet]. 2000 Feb 29 - Identifier NCT03535194, a study to assess if mirikizumab is effective and safe compared to secukinumab and placebo in moderate to severe plaque psoriasis (OASIS-2). Bethesda (MD): National Library of Medicine (US); 2006. Available from: https://clinicaltrials.gov/ct2/show/ NCT03535194?term $=$ mirikizumab\&draw $=2 \&$ rank $=12$. Accessed April 26, 2020.

22. ClinicalTrials.gov [Internet]. 2000 Feb 29 - Identifier NCT03556202, A long-term study to evaluate safety and maintenance of treatment effect of LY3074828 in participants with moderate-to-severe plaque psoriasis (OASIS-3). Bethesda (MD): National Library of Medicine (US); 2006. Available from: https://clinicaltrials.gov/ct2/show/ NCT03556202? term $=$ mirikizumab\&draw $=2 \&$ rank $=21$. Accessed April 26, 2020.

23. Salimi S, Yamauchi PS, Thakur R, et al. Interleukin 23p19 inhibitors in chronic plaque psoriasis with focus on mirikizumab: a narrative review. Dermatol Ther. 2020;33:e13800. doi:10.1111/dth.13800

24. Papp KA, Weinberg MA, Morris A, Reich K. IL17A/F nanobody sonelokimab in patients with plaque psoriasis: a multicentre, randomised, placebo-controlled, phase $2 \mathrm{~b}$ study. Lancet. 2021;397:1564-1575. doi:10.1016/S0140-6736(21)00440-2

25. Wrobleski ST, Moslin R, Lin S, et al. Highly selective inhibition of tyrosine kinase 2 (TYK2) for the treatment of autoimmune diseases: discovery of the allosteric inhibitor BMS-986165. J Med Chem. 2019;62:8973-8995. doi:10.1021/acs.jmedchem.9b00444

26. Papp K, Gordon K, Thaci D, et al. Phase 2 trial of selective tyrosine kinase 2 inhibition in psoriasis. N Engl J Med. 2018;379:1313-1321. doi:10.1056/NEJMoa1806382

27. Cohen S, Barer F, Itzhak I, Silverman MH, Fishman P. Inhibition of IL-17 and IL-23 in human keratinocytes by the A3 adenosine receptor agonist piclidenoson. J Immunol Res. 2018; eCollection:2310970.

28. David M, Akerman L, Ziv M, et al. Treatment of plaque type psoriasis with oral CF101: data from an exploratory randomized phase 2 clinical trial. $J$ Eur Acad Dermatol Veneredol. 2012;3:361-367. doi:10.1111/j.1468-3083.2011.04078.x
29. Mattei PL, Corey KC, Kimball AB. Cumulative life course impairment: evidence for psoriasis. Curr Probl Dermatol. 2013;44:82-90.

30. Gisondi P, Bellinato F, Girolomoni G, Albanesi C. Pathogenesis of chronic plaque psoriasis and its intersection with cardio-metabolic comorbidities. Front Pharmacol. 2020;11:117. doi:10.3389/ fphar.2020.00117

31. Gisondi P, Talamonti M, Chiricozzi A, et al. Treat-to-target approach for the management of patients with moderate-to-severe plaque psoriasis: consensus recommendations. Dermatol Ther (Heidelb). 2021;11:235-252. doi:10.1007/s13555-020-00475-8

32. Sbidian E, Chaimani A, Garcia-Doval I, et al. Systemic pharmacological treatments for chronic plaque psoriasis: a network meta-analysis. Cochrane Database Syst Rev. 2021;19:4.

33. Coates LC, Kavanaugh A, Mease PJ, et al. Group for research and assessment of psoriasis and psoriatic arthritis 2015 treatment recommendations for psoriatic arthritis. Arthritis Rheumatol. 2016;68:1060-1071. doi:10.1002/art.39573

34. Vitae Pharmaceuticals Inc. Vitae Pharmaceuticals achieves proof-ofconcept with first-in-class RORyt inhibitor in moderate to severe psoriasis. Available at: https://www.globenewswire.com/newsrelease/2016/03/16/820582/0/en/Vitae-Pharmaceuticals-AchievesProof-of-Concept-with-First-in-Class-RORyt-Inhibitor-in-Moderateto-Severe-Psoriasis.html. Accessed March 16, 2016.

35. Gege C. ROR $\gamma \mathrm{t}$ inhibitors as potential back-ups for the phase II candidate VTP-43742 from Vitae Pharmaceuticals: patent evaluation of WO2016061160 and US20160122345. Expert Opin Ther Pat. 2017;27(1):1-8. doi:10.1080/13543776.2017.1262350

36. Bell M, Foley D, Naylor C, et al. Discovery of super soft-drug modulators of sphingosine-1-phosphate receptor 1. Bioorg Med Chem Lett. 2018;28:3255-3259. doi:10.1016/j.bmcl.2018.07.044

37. Eliasson L, Bewley AP, Mughal F, et al. Evaluation of psoriasis patients' attitudes toward benefit-risk and therapeutic trade-offs in their choice of treatments. Patient Prefer Adher. 2017;11:353-362. doi:10.2147/PPA.S121838

38. Gisondi P, Geat D, Conti A, et al. TNF- $\alpha$ inhibitors biosimilars as first line systemic treatment for moderate-to-severe chronic plaque psoriasis. Expert Rev Clin Immunol. 2020;16:591-598. doi:10.1080/ 1744666X.2020.1771182

39. Barker J, Girolomoni G, Egeberg A, Goncalves J, Pieper B, Kang T. Anti-TNF biosimilars in psoriasis: from scientific evidence to real-world experience. $J$ Dermatolog Treat. 2020;31:794-800. doi:10.1080/09546634.2019.1610553

40. Puig L, Carrascosa JM, Notario J. Biosimilars in the treatment of psoriasis: an update. Actas Dermosifiliogr. 2020;11:809-814. doi:10.1016/j.ad.2020.03.008

\section{Publish your work in this journal}

Biologics: Targets and Therapy is an international, peer-reviewed journal focusing on the patho-physiological rationale for and clinical application of Biologic agents in the management of autoimmune diseases, cancers or other pathologies where a molecular target can be identified. This journal is indexed on PubMed Central, CAS, EMBase,
Scopus and the Elsevier Bibliographic databases. The manuscript management system is completely online and includes a very quick and fair peer-review system, which is all easy to use. Visit $\mathrm{http}: / / \mathrm{www}$.dovepress.com/testimonials.php to read real quotes from published authors. 